\title{
Formulation and Development of Fixed Dose Combination of Antihypertensive and Antidiabetic Agent for treatment of Co-existent Type Two Diabetes Mellitus and Hypertension
}

\author{
Nisharani Ranpise*, Preeti Jamkar, Harshada Langote \\ Sinhgad College of Pharmacy, Vadgaon (Bk.), Pune-411041 Maharashtra, India
}

\begin{abstract}
Objective: Coexistent Type Two Diabetes Mellitus (TTDM) and hypertension exposes patients to severe co-morbidities. Complex polypharmacy suggested by international organizations leads to the increased pill burden and decreased patient compliance which leads to worsening of conditions. Materials and Methods: Present research aims at the formulation and development of fixed dose combination (FDC) for treatment of coexistent hypertension and TTDM as it will help in increasing patient compliance. Literature survey and patient survey facilitated the selection of metformin $\mathrm{HCl}$, telmisartan, atorvastatin and aspirin for the formulation of FDC. Formulation and development of enteric coated aspirin tablet and the bilayer tablet of metformin $\mathrm{HCl}$, telmisartan \& atorvastatin calcium were two major parts of this research. Aspirin was enteric coated by compression coating technique to avoid gastric irritation. Bilayer tablet separating metformin $\mathrm{HCl}$ layer from the layer of a mixture of telmisartan and atorvastatin calcium was prepared to avoid the physical incompatibility problem between metformin $\mathrm{HCl}$ and telmisartan. The final FDC formulation was prepared by encapsulating, enteric coated aspirin tablet and bilayer tablet in a size 00 capsule and subjected to accelerated stability studies. Results: Batch F2 of bilayer tablet containing one layer of metformin $\mathrm{HCl}$ and the other layer having a mixture of telmisartan\& atorvastatin calcium releases more than $80 \%$ of metformin $\mathrm{HCl}$, telmisartan and atorvastatin calcium within 1 hour. Batch $\mathrm{A} 2 \mathrm{E}$ of enteric coated aspirin tablet was found to show delayed and complete drug release as compared to $A 2 D$ within 3.5 hours. Similarity factor $\mathrm{f} 2$ was found to be 69.33. After long term storage of formulation at $40^{\circ} \mathrm{C} / 75 \% \mathrm{RH}$, stability of drug components remained unaffected. Conclusion: FDC of antihypertensive and antidiabetic agent will be revolutionary in the treatment of coexistent TTDM and hypertension as it will be a cheaper alternative to current therapy.

Key words: Coexistent type two diabetes mellitus and hypertension, Treatment for coexistent type two diabetes mellitus and hypertension, Fixed Dose Combination of Antihypertensive and Antidiabetic Agent, Fixed dose combination.
\end{abstract}

\section{INTRODUCTION}

A deadly combination of coexistent Type Two Diabetes Mellitus and hypertension is influencing the world very rapidly. In developed countries $60-65 \%$ prevalence is seen in the twin epidemic of this 'deadly combination'. Rarely T2DM and hypertension are seen isolated nowadays. This is because diabetic patients are two times more pre- disposed to have hypertension. ${ }^{1}$ In T2DM patients nephropathy and insulin sensitivity (resistance) are the major factors which contribute significantly and independently in the genesis of hypertension. Raised insulin levels in diabetic patients promote sodium retention and along with the sodium glucose also gets reabsorbed. This sodium and fluid retention generates hypervolemia and
Submission Date : 12-09-14 Revision Date : :18-11-14 Accepted Date : :01-12-14

DOI: $10.5530 /$ ijper.48.4s.14 Correspondence Address Dr. (Mrs.) Nisharani S. Ranpise

Professor and H.O.D of Pharmaceutics,

Sinhgad College of Pharmacy,

Vadgaon(Bk.), Pune-411041

Maharashtra, India.

E-mail:nisha_ranpise@

yahoo.com

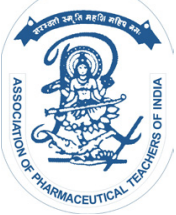

www.ijper.org 
hypertension. ${ }^{2}$ Also, as a consequence of various biochemical phenomena occurring in diabetic individuals such as an increase in the activity of liver lipase leading to lipid abnormalities like increase in VLDL levels, reduction in HDL cholesterol, hypertriglyceridaemia, generation of dense and short LDL particles, hypertension may appear in diabetic individuals. ${ }^{3}$ People $20 \%$ or more with hypertension suffer from diabetes, and $80 \%$ or more with diabetes may develop hypertension.

T2DM and hypertension co-existence exposes patients to severe co-morbidities like microangiopathy (renal, retinal and neural), macroangiopathy (atherosclerosis) and lower limb amputations. ${ }^{4-9}$ So it becomes necessary to treat coexistent T2DM and hypertension in time and with an appropriate drug regimen. The American Diabetes Association Recommends the use of metformin (if tolerated and not contraindicated), ACEI or $\mathrm{ARB}$, any statin along with aspirin (antiplatelet agent) as a first line therapy to maintain the targeted blood glucose level, essential blood pressure control and also to avoid further cardiovascular complications. In some chronic cases intensified therapy with the addition of some more pharmacological agents, e.g. sulphonylurea or GLP 1 agonist or CCB to above mentioned regimen is required. ${ }^{10}$ Such, a complex drug regimen leads to increased pill burden and often reflects into decreased patient compliance. This poor adherence to prescribed regimen, then gets translated into worsening of conditions and increase in hospitalization and all-cause mortality to a significant extent. ${ }^{11-12}$ Noncompliance and lack of persistence with treatment is the major problem associated with the therapy of coexistent hypertension and T2DM. Contributing reasons for this poor adherence may be polypharmacy, complex drug regimen, increased pill burden, cost and the need of long term therapy. A study carried out by Marsha A. Raebel et al. has shown that in patients with a newly ordered medication for hypertension, diabetes or hyperlipidemia 7\% were found primarily non-adherent. ${ }^{13}$ Lower than $80 \%$ compliance was responsible for 1.39 times increased risk of undergoing a modification in the initially prescribed antihypertensive drug regimen. ${ }^{14}$ Similarly a study conducted out by Ho PM et al. demonstrated that significantly higher rates of morbidity and hospitalization, coupled with significantly higher $\mathrm{Hb} \mathrm{A} 1 \mathrm{C}, \mathrm{BP}$, and LDL-C levels were associated with $<80 \%$ adherence to their treatment regimen including oral antidiabetes agents, antihypertensive agents, and/or statin therapy compared with more adherent patients. Importantly, each $25 \%$ improvement in adherence was associated with a reduction in A1C $(0.05 \%)$, systolic BP/diastolic BP (SBP/DBP, 1.0/1.2 $\mathrm{mm} \mathrm{Hg}$ ), and LDL-C (3.8 $\mathrm{mg} / \mathrm{dL})$ that correlated with a significant $(\mathrm{P}<0.1)$ reduc- tion in all-cause hospitalization and mortality. ${ }^{15}$ This non adherence also leads to increased death rate and morbidity. ${ }^{16}$

Patients when switched to FDC from combination therapy shows improved adherence. A study carried out by Melikian $\mathrm{C}$ et al. in diabetic patients demonstrated that adherence to prescribed regimen was increased to $77 \%$ with the use of glyburide and metformin FDC compared to $54 \%$ when these agents were in 2 pills. ${ }^{17}$ Likewise, it was found that compliance was $19 \%$ greater in hypertensive patients taking lisinopril/HCTZ combination compared to drugs taken in 2 different tablets. ${ }^{18}$ Along with improving adherence FDC also lowers the cost involved in the treatment of cardiovascular disease. ${ }^{19}$ Therefore, use of FDC will be one of the promising solution in the treatment of coexistent T2DM and hypertension where patients have to take 4-5 tablets on an average per day and thus show poor compliance to therapy.

The present research aims at the formulation and development of FDC of antihypertensive agent and antidiabetic drugs. Metformin $\mathrm{HCl}$ (insulin sensitizer), telmisartan (ARB), atorvastatin calcium (HMG Co-A reductase inhibitor) and aspirin were selected for the formulation according to a literature review and patient survey conducted in local hospitals. Absence of significant pharmacokinetics as well as pharmacodynamic interactions between these drugs supported their selection. In contrast to it there exist some synergistic interactions between these drugs which further confirmed selection of these agents. For instance co-administration of telmisartan and aspirin in male Sprague-Dawley streptozotocin induced diabetic rats leads to retardation of progress of diabetic nephropathy due to suppression of the augmented TGF $\beta /$ smad signaling. ${ }^{20}$ Metformin and telmisartan co-administration leads to antiobesity effect which in turn will be helpful for diabetic patients. ${ }^{21}$ Statin and ARB co-administration leads to effective prevention of cardiovascular end points. ${ }^{22}$ Metformin and statin combination leads to the enhancement of the antidiabetic activity of metformin as pharmacodynamic interaction between metformin and statin increases bioavailability of metformin and leads to marked decrease in serum glucose level. ${ }^{23}$ So this formulation will be definitely a savior for the patients of coexistent T2DM and hypertension from the pill burden.

\section{MATERIALS AND METHODS}

\section{Materials}

Metformin $\mathrm{HCl}$, telmisartan, atorvastatin calcium, aspirin and Eudragit ${ }^{\circledR} \mathrm{L} 100-55$ were received as gift samples from the Lupin Research Park, Pune. Tablet excipients 
were procured from Merck Specialties Pvt. Ltd, Mumbai. Starch $1500^{\circledR}$ was obtained from Colorcon Asia Pvt. Ltd., Goa. All the reagents used during HPLC analysis were of HPLC grade and purchased from Hi Media Laboratories Pvt. Ltd, Mumbai.

\section{Methods}

\section{Preformulation studies}

\section{Drug-drug and drug-excipients compatibility study}

Pure drugs and physical mixtures of drugs in a 1:1 w/w ratio were prepared in the given combination and kept in screw capped transparent vials for 21 days at $37^{\circ} \mathrm{C}$. After 21 days they were subjected to DSC analysis. The DSC thermograms of pure drugs and mixtures were recorded by using a differential scanning calorimeter (Mettler Toledo DSC-823'e system) equipped with a computerized data station. All samples were weighed and heated at a scanning rate of $10^{\circ} \mathrm{C} / \mathrm{min}$ between 30 and $300^{\circ} \mathrm{C}$ and $60 \mathrm{ml} / \mathrm{min}$ of nitrogen flow. For drugexcipient compatibility study, physical mixtures of drugs in a $1: 1 \mathrm{w} / \mathrm{w}$ ratio with excipients were prepared and kept in screw capped transparent vials for 21 days at $37^{\circ} \mathrm{C}$. After 21 days they were subjected to IR analysis.

\section{Formulation of fixed dose combination of metformin $\mathrm{HCl}$, telmisartan, atorvastatin calcium and aspirin}

\section{Formulation of enteric compression coated aspirin tablet}

\section{Formulation of aspirin core tablet}

The inner core tablet was prepared by direct compression method. The powder mixture of aspirin $(75 \mathrm{mg})$, starch $1500^{\circledR}(5 \mathrm{mg})$ and PVP K 30 (5 mg) were dry blended first for 20 minutes, followed by the addition of stearic acid ( $3 \mathrm{mg})$ and talc $(2 \mathrm{mg})$. Stearic acid was used since magnesium stearate cannot be used in formulations containing aspirin. The powder mixture was further blend for 10 minutes and it was then evaluated for flow properties. The resulting powder mixture was then compressed into tablets (average tablet weight 85 $\mathrm{mg}$ ) using a rotary tablet machine equipped with $6.5 \mathrm{~mm}$ flat faced punch. The core tablets were evaluated for thickness, hardness, weight variation, friability and drug content.

\section{Compression coating of aspirin core tablet}

With the aim of avoiding gastric irritation problem on exposure of aspirin to gastric mucosa, enteric coating was done. Good compressibility and good flow properties of Eudragit ${ }^{\circledR} \mathrm{L} 100-55$ facilitated its use as a coating polymer for compression coating. Different ratios of aspirin: Eudragit ${ }^{\circledR}$ L 100-55 (core: coat ratios) were tried ranging from $1: 1$ to $1: 3$ to avoid the gastric irritation problem associated with aspirin.

Compression coating was done by the direct compression technique using rotary tablet machine with different size punches depending on the weight. Process of compression coating is mainly divided into two parts. The first of which is the slight pre-compression of the first coating layer so as to avoid the sleeping of centrally placed core because of force exerted on it while compression. The second part involves the coating of core with the other layer, achieved by placing the core tablet in the center over the initially compressed coat followed by placing the second part of coating material and subjecting the tablet to the final compression with full compression force. Thus, this forms a sandwiched tablet where, core is sandwiched between the coats.

\section{Selection of core: coat and upper: lower layer weight ratio of coating material}

Aspirin tablet was coated using different ratios of core:coat, i.e. 1:1, 1:2 and 1:3 to formulate a stable compression coated tablet. In these tablets lower and upper layer ratio of coating material was kept constant i.e. 1:1. The concentration which was found to be more stable in acidic $0.1 \mathrm{~N} \mathrm{HCl}$ during a disintegration test was selected for further studies. The selected concentration was used in the different ratios of its layers, i.e. lower layer and upper layer in range of 1:1, 1:2, 1:3. The ratios which showed resistance to degradation in $0.1 \mathrm{~N} \mathrm{HCl}$ during a disintegration test and desired drug release pattern during In vitro dissolution study was used for further evaluation. The above compression coated tablets were evaluated for physical properties like weight variation, thickness, hardness, friability and drug content.

\section{Disintegration test}

The disintegration time of enteric compression coated tablets was determined by using a disintegration test apparatus (Model-ED-2L, Electrolab, India) for six tablets. The apparatus was operated for first $2 \mathrm{hrs}$ in $0.1 \mathrm{~N}$ $\mathrm{HCl}$ without disc and then $0.1 \mathrm{~N} \mathrm{HCl}$ was replaced with $\mathrm{pH} 6.8$ buffer solution and operated for further $1 \mathrm{~h}$ with discs maintained at $37 \pm 2^{\circ} \mathrm{C}$.

\section{Dissolution study of enteric compression coated tablet of aspirin}

Dissolution testing of enteric compression coated aspirin tablet was carried out in triplicate in accordance with the USP 30/NF 25 monograph for aspirin delayed release tablets. Drug release was determined using dissolution apparatus I (baskets) at 100 RPM (DS 8000, Lab India, Mumbai) and $37^{\circ} \mathrm{C} \pm 0.5^{\circ} \mathrm{C}$. During the acid 
Table 1: Composition of bilayer tablet of metformin $\mathrm{HCl}$, telmisartan and atorvastatin calcium.

\begin{tabular}{|l|c|c|}
\hline \multicolumn{1}{|c|}{ Ingredient } & F1 & F2 \\
\hline \multicolumn{2}{|c|}{ Metformin HCl immediate release layer } \\
\hline Metformin HCL & 500 & 500 \\
\hline Starch 1500 & 10 & 10 \\
\hline PVP K 30 & 10 & 10 \\
\hline Avicel PH 101 & 25 & 25 \\
\hline Mg. Stearate & 3 & 3 \\
\hline Talc & 2 & 2 \\
\hline D.Water & q.s. & q.s. \\
\hline Total layer weight & 550 & 550 \\
\hline Atorvastatin calcium and telmisartan & immediate release \\
layer & & \\
\hline Telmisartan & 40 & 40 \\
\hline Atorvastatin calcium & 10 & 10 \\
\hline Meglumine & 16 & 23 \\
\hline Starch 1500 & 10 & 10 \\
\hline PVP K 30 & 10 & 10 \\
\hline MCC & 10 & 3 \\
\hline Mg. Stearate & 5 & 5 \\
\hline Talc & 5 & 50 \\
\hline D.water & q.s. & \\
\hline Total layer weight & 110 & \\
\hline $\begin{array}{l}\text { Total weight of } \\
\text { bilayer tablet }\end{array}$ & 660 & \\
\hline (All quantities are in mg) & & \\
\hline
\end{tabular}

stage (2 hours in $0.1 \mathrm{~N} \mathrm{HCl}$ ) aliquots were withdrawn at defined time intervals and tested for content of aspirin released by a UV spectrophotometer (UV 1800 Shimadzu, Japan) at $280 \mathrm{~nm}$. The specification for the acid phase is not more than $10 \%$ aspirin dissolved. The acid was then drained from the vessel, and replaced with $\mathrm{pH}$ 6.8 phosphate buffer. Sample aliquots were withdrawn from the buffer phase in 30, 45, 60 and 90 minutes, and analyzed for the amount of aspirin dissolved by a UV spectrophotometer (UV 1800 Shimadzu, Japan) at 267 $\mathrm{nm}$ (Isobestic point of aspirin and salicylic acid). The specification for the buffer phase is not less than $80 \%$ drug dissolved after 90 minutes. The cumulative percentage drug release was calculated. Best formulation was compared with conventional marketed product and similarity factor $f_{2}$ value was determined.

\section{Formulation of bilayer tablet of metformin $\mathrm{HCl}$,} telmisartan and atorvastatin calcium

\section{A) Preparation of immediate release metformin} HCl layer

Immediate release metformin $\mathrm{HCl}$ layer was prepared by the aqueous granulation method. The required materials (Table 1) were passed through \# 40 sieve. All materials, except polyvinyl
Pyrrolidone K 30 (PVP K 30) and lubricants were thoroughly mixed in a polybag for $30 \mathrm{~min}$ and then granulated using PVP as a binder solution. The wet granules were passed through \# 14 sieve. The granules prepared were dried at $60^{\circ} \mathrm{C}$ for $5 \mathrm{~h}$ in a tray dryer. The dried granules were then passed through \# 20 sieve. To this, the lubricants which were previously passed through \# 40 sieve were added and mixed for $2 \mathrm{~min}$.

B) Preparation of immediate release layer of telmisartan and atorvastatin calcium.

An immediate release layer of telmisartan and atorvastatin calcium was prepared by aqueous and direct compression method. The required materials (Table 1) were passed through \# 40 sieve. All materials, except polyvinyl pyrrolidone (PVP), meglumine and lubricants were thoroughly mixed in a polybag for $30 \mathrm{~min}$ and then granulated using a solution of PVP K 30 (10\%) and meglumine. The wet granules were passed through \# 14 sieve. The granules prepared from aqueous granulation were dried at $60^{\circ} \mathrm{C}$ for $5 \mathrm{~h}$ in a tray dryer. The dried granules were then passed through \# 20 sieve. To this, the lubricants which were previously passed through \# 40 sieve were added and mixed for $2 \mathrm{~min}$. For direct compression method all the required materials, except lubricants were weighed accurately (Table 1) and mixed thoroughly in mortar using pestle. Lubricants were added towards the end and mixed well. The formed poweder mixture was then used further for preparing bilayer tablet,separating metformin $\mathrm{HCl}$ layer from the layer of a mixture of telmisartan and atorvastatin calcium.

\section{Compression of bilayer tablet of FDC of metformin $\mathrm{HCl}$, telmisartan and atorvastatin calcium}

The tablets of $\mathrm{F} 1$ and $\mathrm{F} 2$ were compressed using a rotary tablet machine equipped with $17 \times 6 \mathrm{~mm}$ capsule shape standard concave punch. First a slight pre-compression was made with the metformin $\mathrm{HCl}$ layer so that the layer was uniformly distributed. After that, the second layer of atorvastain calcium and telmisartan was added and the final compression was made. Parameters such as hardness, weight variation, friability, thickness, content uniformity were evaluated for the prepared tablets. For the purpose of selecting the batch for the dissolution and further study a tablet was crushed and dissolved in $900 \mathrm{ml}$ of $0.1 \mathrm{~N} \mathrm{HCl}$. After the complete dissolution of tablet, $\mathrm{pH}$ of the solution was checked. Depending on the attained $\mathrm{pH}$ value batch was selected for further dissolution study.

\section{In vitro dissolution studies}

The dissolution test for bilayer tablet was performed in triplicate using a USP type II (paddle) dissolution apparatus (DS 8000, Lab India, Mumbai) at $37^{\circ} \mathrm{C} \pm$ 


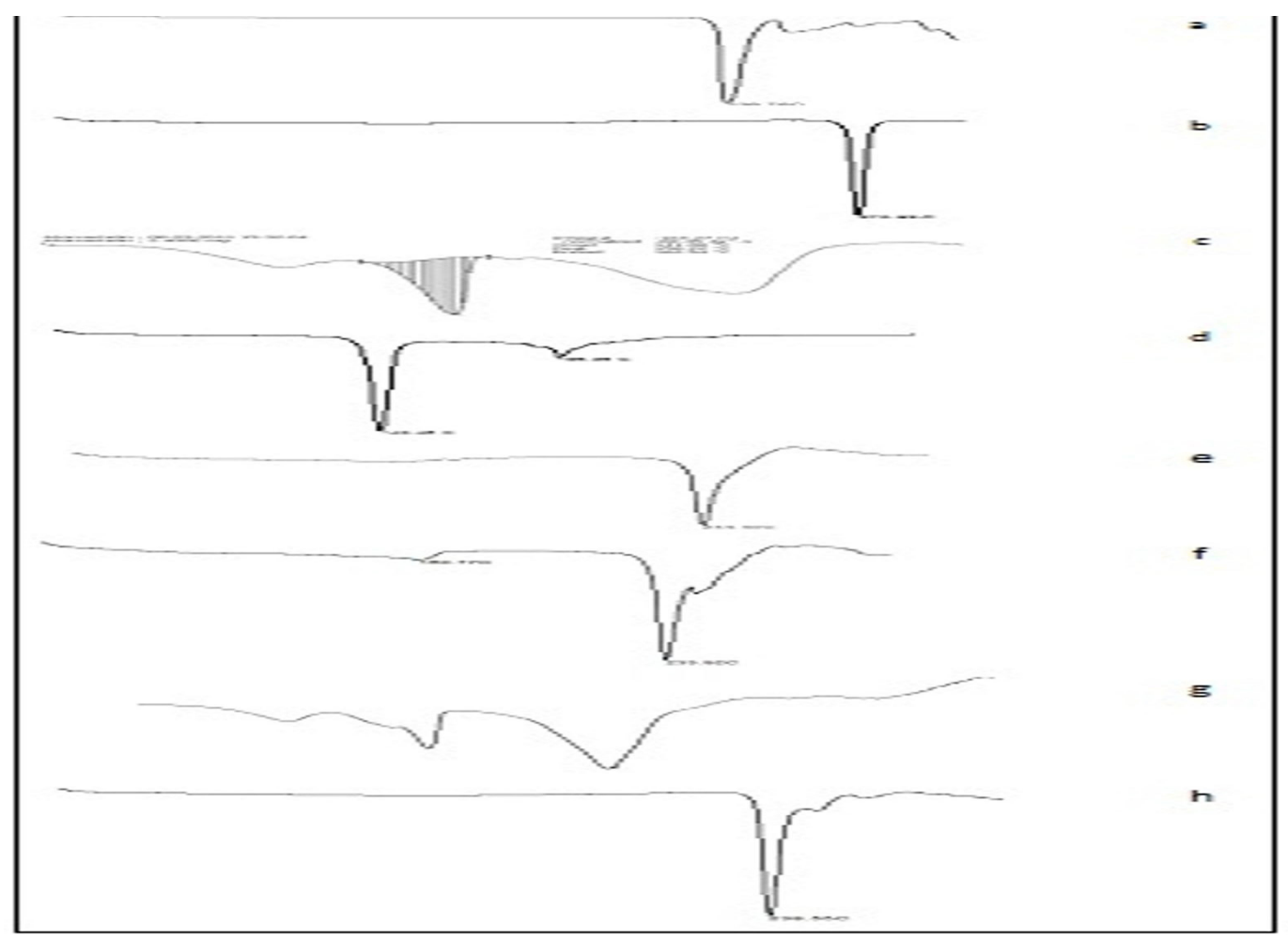

Figure1: DSC thermograms of drugs and various physical mixtures.

a) Pure metformin $\mathrm{HCl}$ b) Telmisartan c) Atorvastatin calcium d) Aspirin e) Mixture of metformin $\mathrm{HCl}$, telmisartan and atorvastatin calcium f) Mixture of metformin $\mathrm{HCl}$ and atorvastatin calcium g) Mixture of telmisartan and atorvastatin calcium h) Mixture of metformin $\mathrm{HCl}$ and telmisartan

$0.5^{\circ} \mathrm{C}$ and $50 \mathrm{RPM}$ speed in $\mathrm{pH} 6.8$ phosphate buffer for 1 hour. Aliquots of dissolution fluid were removed at specified time intervals $(10 \mathrm{~min})$ and assayed for the release of metformin $\mathrm{HCl}$, telmisartan and atorvastatin by a validated HPLC method. At each time of withdrawal, $10 \mathrm{ml}$ of fresh corresponding dissolution medium was replaced into the dissolution flask.

\section{Stability studies}

The FDA and ICH specify the guidelines for stability testing of new drug products, as a technical requirement for the registration of pharmaceuticals for human use. The study was performed under accelerated stability conditions at $40^{\circ} \mathrm{C} \pm 2{ }^{\circ} \mathrm{C} / 75 \% \mathrm{RH} \pm 5 \% \mathrm{RH}$ for 3 months.

\section{RESULT AND DISCUSSION}

\section{Drug-drug and drug-excipients compatibility study}

The DSC thermogram of pure drugs and physical mixtures are shown in the (Figure 1). The DSC thermogram of pure metformin $\mathrm{HCl}$ (a) indicated a sharp endothermic peak at 236 (C corresponding to melting of pure metformin $\mathrm{HCl}$. The thermogram of pure telmisartan (b) indicated a sharp endothermic peak at $270^{\circ} \mathrm{C}$ corresponding to melting of pure telmisartan and the thermogram of pure atorvastatin calcium (c) indicated a sharp endothermic peak at $160^{\circ} \mathrm{C}$ corresponding to melting of pure atorvastatin calcium. The thermogram of pure aspirin (d) indicated a sharp endothermic peak at $141^{\circ} \mathrm{C}$ corresponding to melting of pure aspirin.

The peak for telmisartan was vanishedin a mixture of metformin $\mathrm{HCl}$, telmisartan and atorvastatin calcium (e) as well as in a mixture of metformin $\mathrm{HCl}$ and telmisartan (f) also peak of telmisartan was not observed. This indicated the presence of interaction between these components. In a mixture of metformin $\mathrm{HCl}$ and atorvastatin calcium ( $\mathrm{f}$ ) as well as in a mixture of atorvastatin calcium and telmisartan $(\mathrm{g})$ peaks of both the drugs were present, but peak broadening was observed and also the relative intensities are changed due to dilution of the drug in the samples. So from DSC study it can be concluded that metformin $\mathrm{HCl}$ and telmisartan are physically incompatible with each other. Also telmisartan and atorvastatin calcium did not form a complex with each other as the endothermic peaks remained unchanged in position. Similarly metformin $\mathrm{HCl}$ and atorvastatin also found to be compatible with each other as their endothermic peaks remained unchanged in position.

The IR spectra of pure drugs and physical mixture of all drugs along with all excipients are shown in (Figure 2). The absence of significant change in the IR specta indicates the physical compatibility between drug and excipients. 


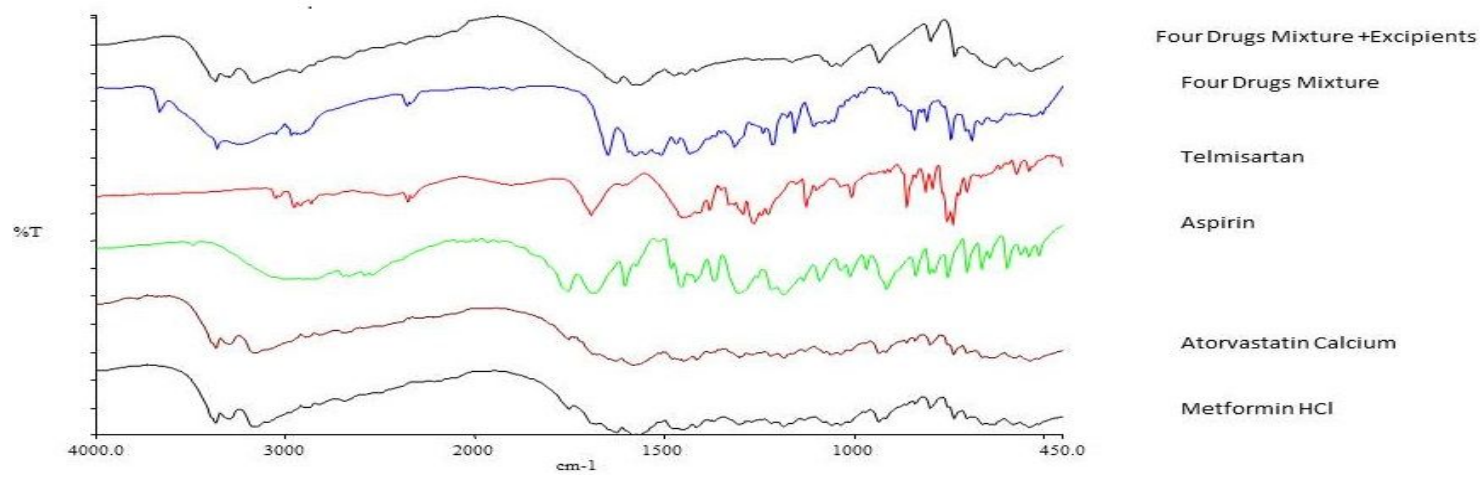

Figure 2: IR spectra of drugs, physical mixture of drugs and physical mixture of drugs with excipients

\section{Formulation and development of FDC of metformin $\mathrm{HCl}$, telmisartan, atorvastatin calcium and aspirin}

From the DSC studies, we concluded that telmisartan and metformin $\mathrm{HCl}$ are not physically compatible with each other. Therefore, we went for preparing the bilayer tablet of metformin $\mathrm{HCl}$, telmisartan and atorvastatin calcium separating metformin $\mathrm{HCl}$ layer from the layer of a mixture of telmisartan and atorvastatin.

As it is known aspirin leads to irritation to gastric mucosa, it is necessary to avoid its release in the gastric environment. Therefore, aspirin was enteric coated.

Then bilayer tablet of metformin $\mathrm{HCl}$, telmisartan and atorvastatin calcium separating metformin layer from a layer of a mixture of telmisartan and atorvastatin calcium and enteric compression coated aspirin tablet were further encapsulated in a size 00 hard gelatine capsule.

\section{Evaluation of core aspirin tablet}

Weight variation $(85.33 \pm 0.577 \mathrm{mg})$, thickness $(3 \pm$ $0.06)$ and hardness $(5 \pm 0.577)$ of the core tablets $(\mathrm{P})$ were found to be within acceptable limits. The friability was $\leq 1 \%$ for all the formulations, which is an indication of good mechanical resistance of the tablet. The core tablets completely disintegrated within 5 min. Drug content of core tablets was observed within the range $97.26-98.56 \%$. The In vitro release of aspirin from core tablet was $98.24 \%$ within 5 min in $\mathrm{pH} 6.8$ phosphate buffer medium.

\section{Selection of core: coat and upper: lower weight ratio of coating material}

Formulations A1, A2 and A3 subjected to disintegration study as per USP. Formulation A1 was unable to protect core tablet, A2 and A3 were stable for the first $2 \mathrm{~h}$ in 0.1 $\mathrm{N} \mathrm{HCl}$ and starts to rupture after $25 \mathrm{~min}$ and $36 \mathrm{~min}$ in pH 6.8 buffer respectively (Table 2).

Batch A2 was further processed to determine the upper $\left(2^{\text {nd }}\right)$ : lower $\left(1^{\text {st }}\right)$ layer weight ratio of coating material because when the performance of batch $\mathrm{A} 2$ and $\mathrm{A} 3$ in disintegration test were compared, batch A2 tablet was found to show slow disintegration behavior as compared to batch A3 tablet. At the same time as mentioned earlier, it is required to encapsulate the coated tablet in a capsule. So even though batch A3 did not show any signs of coat rupture, the batch was excluded from further studies since its size was large which could not be encapsulated in the size 00 capsule (the higher limit size capsule for human consumption).

The coats of formulation A2 were further modified using different upper: lower layer weight ratios of coating material and subjected to disintegration. Among all formulations A2F fail to protect core tablet as it disintegrates in $0.1 \mathrm{~N} \mathrm{HCl}$ as shown in (Table 3). Formulations $\mathrm{A} 2 \mathrm{D}$ and $\mathrm{A} 2 \mathrm{E}$ were stable in $0.1 \mathrm{~N} \mathrm{HCl}$ and start to disintegrate in $\mathrm{pH} 6.8$ buffer within $30 \mathrm{~min}$. All stable formulations (A2D and A2E) were selected for further study (Table 3).

\begin{tabular}{|c|c|c|c|c|}
\hline \multicolumn{5}{|c|}{ Table 2: Selection of polymer concentration on the basis of disintegration test. } \\
\hline \multicolumn{2}{|c|}{ Formulation } & Punch Size $\mathbf{( m m )}$ & Core: coat ratio & Disintegration Test \\
\hline Code & 170 & 7 & $1: 1$ & $\begin{array}{c}\text { Coat ruptures within } 45 \mathrm{mins} \text { in acidic } \mathrm{pH} \\
1.2 \mathrm{medium} .\end{array}$ \\
\hline & 250 & 7 & $1: 2$ & $\begin{array}{c}\text { Coat remains intact in acidic } \mathrm{pH} 1.2 \mathrm{buffer} \\
\text { medium and ruptures within } 25 \mathrm{~min} \text { in } \mathrm{pH} \\
6.8 \text { buffer medium. }\end{array}$ \\
\hline A2 & 335 & 8 & $1: 3$ & $\begin{array}{c}\text { Coat remains intact in acidic } \mathrm{pH} 1.2 \text { medium } \\
\text { and ruptures within } 36 \mathrm{~min} .\end{array}$ \\
\hline
\end{tabular}


Table 3: Selection of concentration of different layers of compression coat on the basis of disintegration test.

\begin{tabular}{|c|c|c|c|c|c|c|}
\hline \multirow{2}{*}{\multicolumn{2}{|c|}{ Formulation }} & \multirow{2}{*}{$\begin{array}{l}\text { Layer } \\
\text { Ratio }\end{array}$} & \multirow{2}{*}{$\begin{array}{l}\text { Upper } \\
\text { layer }\end{array}$} & \multirow{2}{*}{$\begin{array}{l}\text { Lower } \\
\text { layer }\end{array}$} & \multicolumn{2}{|c|}{ Disintegration Test } \\
\hline & & & & & PBS pH 1.2 & PBSpH 6.8 \\
\hline \multirow{3}{*}{ A2 } & $\mathrm{D}$ & $1: 1$ & 82.5 & 82.5 & No signs of rupture & Coat ruptures after $16 \mathrm{~min}$ \\
\hline & $\mathrm{E}$ & $1: 2$ & 56 & 112 & No signs of rupture & Coat ruptures after $24 \mathrm{~min}$ \\
\hline & $\mathrm{F}$ & $1: 3$ & 42.5 & 127.5 & Coat ruptures after $16 \mathrm{~min}$ & --- \\
\hline
\end{tabular}

\begin{tabular}{|c|c|c|c|c|c|}
\hline \multicolumn{7}{|c|}{ Table 4: Evaluation of compression coated aspirin tablet. } \\
\hline Formulation code & $\begin{array}{c}\text { Hardness } \\
\text { (kg/cm sq.) }\end{array}$ & $\begin{array}{c}\text { Thickness } \\
(\mathbf{m m})\end{array}$ & $\begin{array}{c}\text { Weight } \\
\text { variation }\end{array}$ & $\begin{array}{c}\text { \% Friability } \\
\text { Content uniformity } \\
(\%)\end{array}$ \\
\hline A2D & $6.6 \pm 0.10$ & $5 \pm 0.04$ & $170 \pm 0.05$ & $1.08 \pm 0.05$ & $97.85 \pm 0.08$ \\
\hline A2E & $6.8 \pm 0.05$ & $5 \pm 0.05$ & $170 \pm 0.03$ & $1.03 \pm 0.05$ & $98.96 \pm 0.02$ \\
\hline A2F & $6.8 \pm 0.09$ & $5 \pm 0.07$ & $170 \pm 0.03$ & $1.08 \pm 0.06$ & $97.96 \pm 0.05$ \\
\hline
\end{tabular}

Evaluation of enteric compression coated aspirin tablet:

All physical parameters were found to be within acceptable limit for enteric compression coated aspirin tablets (Table 4). The friability was $\leq 1 \%$ for all the formulations, which is an indication of good mechanical resistance of the tablet. Drug content of tablets was observed within the range $97.85-98.96 \%$ as shown in (Table 4).

\section{In-vitro dissolution study for enteric compression coated aspirin tablet}

In vitro dissolution study for compression coated tablets was carried out in $0.1 \mathrm{~N} \mathrm{HCl}$ for the first $2 \mathrm{~h}$ and continued in phosphate buffer $\mathrm{pH} 6.8$ for $1.5 \mathrm{~h}$. The varying core: coat and upper: lower layer weight ratio in tablet formulations showed a marked difference in their drug release pattern as shown in (Figure 3). Batch A2D showed acid resistance for the first $2 \mathrm{~h}$ but in acid phase drug release was more than $10 \%$, which is unacceptable according to the limits given in USP 30/NF 25. Therefore, both the batches A2D rejected. Batch A2E was found to show delayed and complete drug release as compared to A2D within $3.5 \mathrm{~h}$. Therefore, batch A2E was found to be better and it was selected for further comparison with marketed formulation.

(Figure 4) shows that the drug release pattern from the batch $\mathrm{A} 2 \mathrm{E}$ was approximately same as that of the conventional marketed tablet. Similarity factor F2 was found to be 69.33. Thus, A2E formulation can be considered as showing similar dissolution profile as that of reference products.

\section{Formulation and development of bi-layer tablet of metformin $\mathrm{HCl}$, telmisartan and atorvastatin calcium}

As mentioned in the Table 1addition of meglumine was done to adjust the $\mathrm{pH}$ of the formulation after dissolution. It was needed for maintaining the stability of Atorvastatin calcium in acidic medium. Atorvastatin calcium is given in hydroxy acid form. But in acidic medium it gets converted into an inactive lactone form. The equilibrium of this reaction is obtained within the $\mathrm{pH}$ range of 5.5-6. So meglumine was selected as an alkalizing agent. Conventionally calcium carbonate was used as an alkalizer in atorvastatin formulations, but it may get degraded and produces by products like calcium oxide and $\mathrm{CO} 2$, which may lead to further complications as well as its addition may also hamper tableting process. So use of calcium carbonate was avoided by us. ${ }^{24}$

For the preparation of a layer of a mixture of telmisartan and atorvastatin calcium direct compression technique was preferred over the wet granulation technique as during wet granulation, tablet blend was found to get liquefied leading to failure of granulation. So the option of wet granulation technique to prepare bilayer tablet was dropped at the initial stage only.

\section{Evaluation of bilayer tablet of metformin $\mathrm{HCl}$, telmisartan and atorvastatin calcium}

Weight variation, thickness and hardness of the bilayer tablets (P) were found to be within acceptable lim-

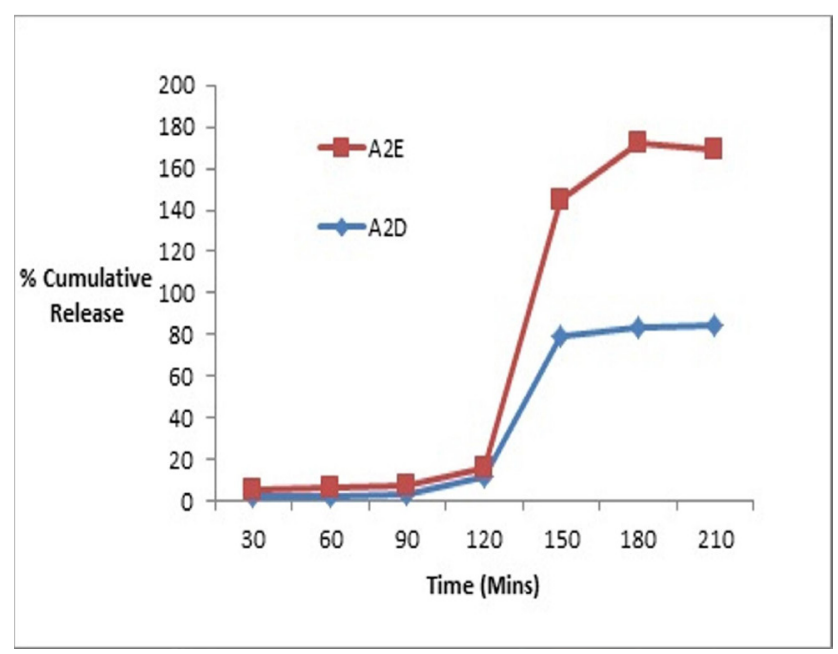

Figure 3: Comparison of dissolution profiles of batch A2 tablets of enteric compression coated aspirin tablets. marketed formulation. 


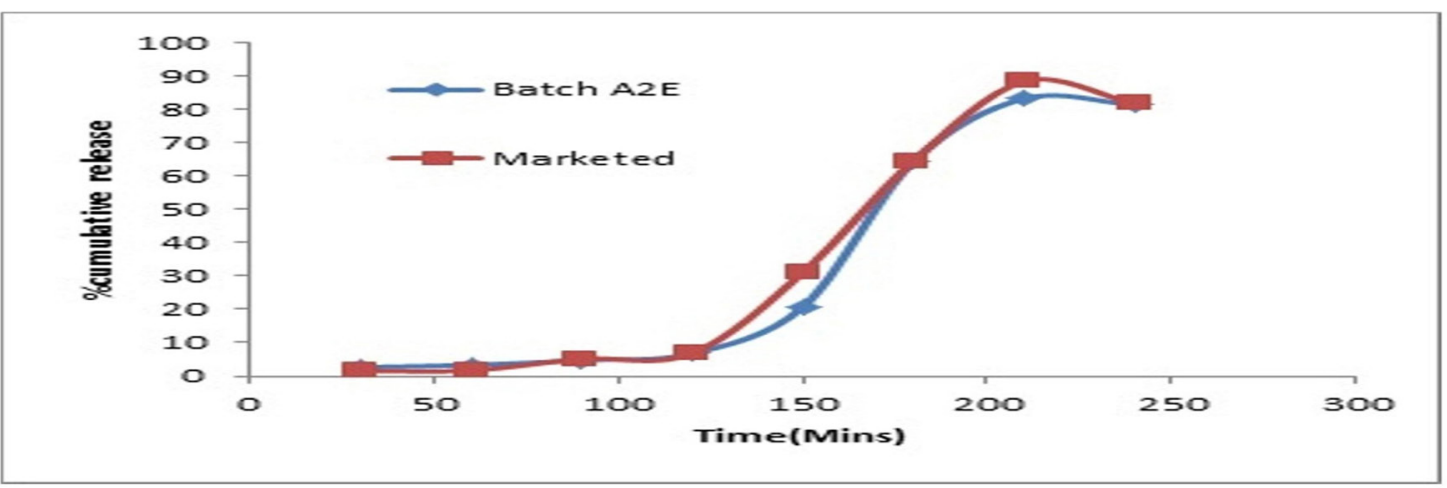

Figure 4: Comparison of dissolution profile of batch A2E of enteric compression coated aspirin tablet with marketed formulation.

\begin{tabular}{|c|c|c|c|c|c|}
\hline \multicolumn{7}{|c|}{ Table 5: Evaluation bilayer tablet. } \\
\hline Formulation code & $\begin{array}{c}\text { Hardness(kg/cm } \\
\text { sq.) }\end{array}$ & Thickness $(\mathbf{m m})$ & $\begin{array}{c}\text { Weight Variation } \\
\text { (mg) }\end{array}$ & \% Friability & Content uniformity \\
\hline F1 & $8 \pm 0.28$ & $7.4 \pm 0.05$ & $661 \pm 0.3$ & $1.35 \pm 0.005$ & $95.88 \pm 0.08$ \\
\hline F2 & $8.5 \pm 0.15$ & $7.3 \pm 0.05$ & $660 \pm 0.4$ & $1.39 \pm 0.005$ & $94.96 \pm 0.02$ \\
\hline
\end{tabular}

its (Table 5). The friability was $\leq 1 \%$ for all the formulations, which is an indication of good mechanical resistance of the tablet. The bilayer tablets completely disintegrated within $8 \mathrm{~min}$. Drug content of core tablets was observed within the range 95.88-94.96 for batch F1 and $\mathrm{F} 2$ as shown in (Table 5).

When the tablets were crushed and dissolved in $\mathrm{pH} 1.2$ buffer, $\mathrm{pH}$ of the solution was shifted to $\mathrm{pH} 4$ in batch $\mathrm{F} 1$ while to $\mathrm{pH} 6$ in batch $\mathrm{F} 2$. As mentioned earlier $\mathrm{pH}$ of 5.5-6 is required for maintaining the stability of atorvastatin calcium in acidic medium, therefore batch F2 was selected for further studies.

\section{In vitro dissolution test for bi-layer tablet of metformin $\mathrm{HCl}$, telmisartan and atorvastatin calcium}

Dissolution profile of batch F2 is shown in (Figure 5). Batch $\mathrm{F} 2$ releases more than $80 \%$ of metformin $\mathrm{HCl}$, telmisartan and atorvastatin calcium within 1 hour.

\section{Stability Study}

After long term storage of formulation at $40^{\circ} \mathrm{C} / 75 \%$ $\mathrm{RH}$, stability of drug components remained unaffected. Drug content of the formulation after stability study is analyzed by a validated HPLC method and it found to be unhampered as shown in (Table 6). Similarly drug release profiles also remained unaffected without any significant changes.

\section{CONCLUSION}

To avoid the problem of poor patient adherence associated with the long term therapy of coexistent T2DM and hypertension formulation of FDC of antihypertensive and oral hypoglycemic agent was developed. Selection of metformin $\mathrm{HCl}$, telmisartan, atorvastatin calcium and aspirin was favored by patient and literature survey. Final formulation consisted of enteric compression coated aspirin tablet and the bilayer tab-

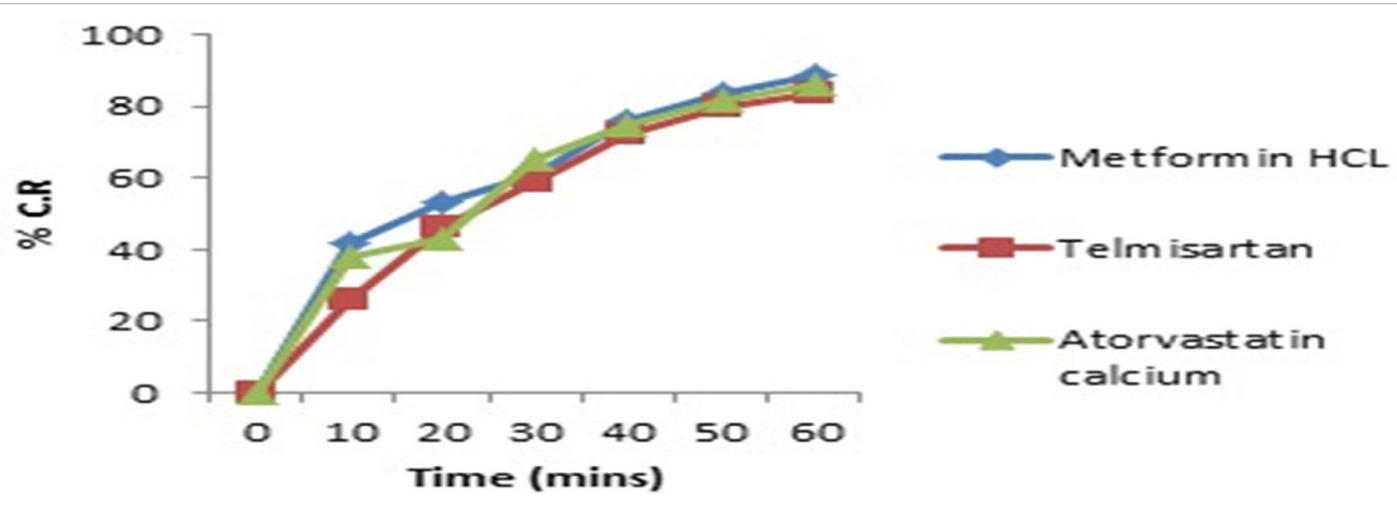

Figure 5: Dissolution profile of batch F2 of bilayer tablet 


\begin{tabular}{|c|c|c|c|c|}
\hline \multirow{2}{*}{ Drug } & \multicolumn{4}{|c|}{ Table 6: Drug content stability study } \\
\cline { 2 - 5 } & on 0 day & on $\mathbf{3 0}$ day & on $\mathbf{6 0}$ day & on 90 day \\
\hline Metformin $\mathrm{HCl}$ & 96.92 & 96.61 & 96.45 & 96.42 \\
\hline Telmisartan & 94.92 & 94.62 & 94.53 & 94.49 \\
\hline $\begin{array}{c}\text { Atorvastatin } \\
\text { calcium }\end{array}$ & 96.92 & 96.92 & 96.92 & 96.92 \\
\hline Aspirin & 98.85 & 98.61 & 98.45 & 97.82 \\
\hline
\end{tabular}

let of metformin $\mathrm{HCl}$, telmisartan and atorvastatin calcium encapsulated in size 00 capsule. The in vitro drug release study revealed that all the drug components were released in desired manner. Thus we can conclude that FDC of antihypertensive and antidiabetic drug was developed successfully.

\section{ACKNOWLEDGEMENT}

We are grateful towards Lupin Reseach Park, Pune, for providing us the gift samples of metformin $\mathrm{HCl}$, atorvastatin calcium, telmisartan and aspirin. We would also like to acknowledge Colorcon Asia, Goa, with a sense of gratitude for providing us Starch 1500, as gift sample.

\section{REFERENCES}

1. Marks J, Raskin P, Nefropatia E. Hypertension en la diabetes. Clin Med de NA 1998; 4: 817-44.

2. Marre M, Berrut G, Bouhanick B. Hypertension and diabetes mellitus. Biomedicine and Pharmacotherapeutics. 1993; 47(2): 61-6.

3. Contreras F, Rivera M, Vasquez J. Diabetes and hypertension pathophysiology and therapeutics. Journal of Human Hypertension. 2000; 14 (1): S26-S31.

4. Epstein, Murray, Sowers J. Diabetes mellitus and hypertension. Hypertension 1992; 19(5): 403- 18.

5. Tedesco M, Natale F, Di Salvo G, Caputo S, Capasso M, Calabro R. Effects of coexisting hypertension and Type 2 diabetes mellitus on arterial stiffness. Journal of Human Hypertension. 2004; 18(7): 469-73.

6. Wong, Evelyn, Backholer K, Gearon E et.al. Diabetes and risk of physical disability in adults: A systematic review and meta-Analysis. Lancet Diabetes Endocrinology. 2013; 1(2): 106-14.

7. Merel J, Luitse A, Biessels G, Guy E, Rutten H, Kappelle L. Diabetes, Hyperglycaemia, and acute ischaemic stroke. Lancet Neurology . 2012; 11(3): 261-71.

8. Gleissner C, Galkina E, Nadler J, Ley K. Mechanisms by which diabetes increases cardiovascular disease. Drug Discovery Today: Disease Mechanisms, Cardiovascular Diseases. 2007; 4(3): 131-40.

9. Today. Study group rapid rise in hypertension and nephropathy in youth with Type 2 Diabetes. Diabetes Care. 2013; 36(6): 1735-41.
10. American Diabetes Association. Standards of medical care in diabetes 2013 Diabetes Care. 2013; 36 (1): S11.

11. Benford M, Milligan G, Pike J, Anderson P, Piercy J, Fermer S. Fixed dose combination Antidiabetic Therapy: Real-World factors associated with prescribing choices and relationship with patient satisfaction and compliance. Advance Therapeutics. 2012; 29(1): 26-40.

12. Chobanian AV. Impact of nonadherence to antihypertensive therapy. Circulation. 2009; 120(16): 1558-60.

13. Raebel M, Ellis J, Carroll N, Bayliss E A, McGinnis B, Schroeder EB. et.al. Characteristics of patients with primary non-adherence to medications for hypertension, diabetes, and lipid disorders. Journal of General Internal Medicine. 2011; 27(1): 57-64.

14. Boris $L$, Van $W$, Klungel $O$. The association between compliance with antihypertensive drugs and modification of antihypertensive drug regimen. Journal of Hypertension 2004; 22(9):1831-37.

15. Ho P, Rumsfeld J, Masoudi F, McClure L, Plomondon E, Steiner F. et al. Effect of medication nonadherence on hospitalization and mortality among patients with diabetes mellitus. Archives of Internal Medicine. 2006; 166(17): 1836-41.

16. Bailey $C$, Kodack M. Patient adherence to medication requirements for therapy of type 2 diabetes. International Journal of Clinical Practice. 2011; 65(3): 314-22.

17. Melikian C, White T, Vanderplas A, Dezii C, Chang, E. Adherence to oral antidiabetic therapy in a managed care organization: a comparison of monotherapy, combination therapy, and fixed-dose combination therapy. Clinical Therapeutics. 2002; 24(3): 460-7.

18. Dezii C. A retrospective study of persistence with single-pill combination therapy vs. Concurrent two-pill therapy in patients with hypertension. Managed Care. 2000; 9(9): S2-S6.

19. Taylor A, Shoheiber O. Adherence to antihypertensive therapy with fixed-dose amlodipine besylate/benazepril $\mathrm{HCl}$ versus comparable component-based therapy. Congestive Heart Failure. 2003; 9(6): 324-32.

20. Mulay S, Gaikwad A, Tikoo K. Combination of aspirin with telmisartan suppresses the augmented TGF $\boldsymbol{\beta}$ /smad signaling during the development of streptozotocininduced type I diabetic nephropathy. Chemico-Biological Interactions. 2010; 185(2): 137-42.

21. Goyal A, Goyal R, Sharma P, Malik Z. Beneficial effect of combination of metformin and telmisartan on high fat diet-induced obesity in Wistar rats. Asian Journal of Pharmaceutics and Clinical Research. 2011; 4(4): 106-11.

22. Nickenig G. Should Angiotensin II Receptor Blockers And Statins Be Combined? Circulation. 2004; 110(8): 1013-20.

23. Anitha N, Rao JV, Kavimani S, Himabindu V. Pharmacodynamic Drug Interaction of Metformin with Statin In Rats. Journal of Pharmacology and Toxicology. 2008; 3(5): 409-13.

24. Mukharya A, Chaudhary S, Patel N, Mansuri N, Misra A. Stable and Bio-equivalent Formulation of HMG-CoA reductase inhibitor: Atorvastatin Calcium. International Journal of Pharmaceutical Sciences Letters. 2011; 2(1): 12-20. 